\title{
Role of Kenae/CCDC125 in cell motility through the deregulation of RhoGTPase
}

\author{
NATSUMI ARAYA $^{1 *}$, HITOSHI ARIMURA ${ }^{2 *}$, KO-ICHI KAWAHARA ${ }^{3}$, NAOKO YAGISHITA $^{1}$, JUNJI ISHIDA ${ }^{4}$, \\ RYOJI FUJII ${ }^{1}$, SATOKO ARATANI ${ }^{1}$, HIDETOSHI FUJITA ${ }^{1}$, TOMOO SATO ${ }^{1}$, YOSHIHISA YAMANO ${ }^{1}$, \\ ITSURO HIGUCHI ${ }^{2}$, MITSUHIRO OSAME ${ }^{2}$, KUSUKI NISHIOKA ${ }^{1}$, AKIYOSHI FUKAMIZU ${ }^{4}$, \\ KIMIYOSHI ARIMURA ${ }^{3}$, IKURO MARUYAMA ${ }^{3}$ and TOSHIHIRO NAKAJIMA ${ }^{1}$

\begin{abstract}
${ }^{1}$ Department of Genome Science, Institute of Medical Science, St. Marianna University School of Medicine,
2-16-1 Sugao Miyamae-ku, Kawasaki, Kanagawa 216-8512; Departments of ${ }^{2}$ Neurology and Geriatrics,

Kagoshima University, 8-35-1 Sakuragaoka, Kagoshima 890-8520; ${ }^{4}$ Center for Tsukuba Advanced

Research Alliance, University of Tsukuba, 1-1-1 Tennodai, Tsukuba 305-8572, Japan
\end{abstract} \\ Faculty of Medicine and ${ }^{3}$ Laboratory and Vascular Medicine, Graduate School of Medical and Dental Sciences,
}

Received May 25, 2009; Accepted July 10, 2009

DOI: 10.3892/ijmm_00000271

\begin{abstract}
Isaac's syndrome is a movement disorder characterized by hyperexcitability of peripheral motor nerves. Patients with Isaac's syndrome often develop auto-antibodies to voltage-gated potassium channels (VGKCs) which block their function. However, anti-VGKC antibodies are not detected in all patients with Isaac's syndrome, suggesting the existence of another etiology. In this study, we performed immunoscreening using the serum from a patient with Isaac's syndrome and identified the novel gene named Kenae/ CCDC125. Expression analysis of Kenae/CCDC125 revealed that its transcript was highly expressed in tissues associated with the immune system, such as the thymus, spleen and bone marrow. In cells stably expressing Kenae/CCDC125, delay in cell motility and deregulation of RhoGTPase (RhoA, Rac1 and cdc42) activity to extracellular stimuli were demonstrated. These results suggest that the novel gene, Kenae/CCDC125, acts as a regulator of cell motility through RhoA, Rac1 and cde42.
\end{abstract}

\section{Introduction}

Isaac's syndrome, the most well-known disease of neuromyotonia, is characterized by the presence of spontaneous

Correspondence to: Dr Toshihiro Nakajima, Department of Genome Science, Institute of Medical Science, St. Marianna University School of Medicine, 2-16-1 Sugao Miyamae-ku, Kawasaki, Kanagawa 216-8512, Japan

E-mail: nakashit@marianna-u.ac.jp

${ }^{*}$ Contributed equally

Key words: Isaac's syndrome, Kenae/CCDC125, cell motility, RhoGTPase and continuous muscle fiber activity (1). Patients present symptoms caused by spontaneous bursts of nerve discharges such as i) pseudomyotonia (slow relaxation) induced by muscle contraction, ii) increased cramping and iii) excessive sweating (2). Recently, it was found that anti-voltage-gated potassium channel (VGKC) antibodies are present in the sera of patients with Isaac's syndrome, and their symptoms respond to immunotherapy (3-6), suggesting that this disorder is caused by an autoimmune mechanism. Furthermore, Isaac's syndrome often occurs concomitantly with other autoimmune conditions, such as myasthenia gravis which is caused by anti-acetylcholine receptor antibodies. However, antibodies against VGKCs are not detected in all cases of Isaac's syndrome. Therefore, we hypothesized that there may be other factors or molecules associated with this disease. In this study, we executed immunoscreening using the serum of a patient with Isaac's syndrome. In the reactive cDNA clones, we identified a novel gene named Kenae/CCDC125. mRNA expression of Kenae/CCDC125 was highly expressed in organs of the immune system, such as the thymus, spleen and bone marrow. In addition, to investigate the molecular function of Kenae/CCDC125, we established HEK293 cells stably expressing this gene and revealed that Kenae/CCDC125 was associated with cell movement.

Therefore, the aim of our study was to ascertain the role of Kenae/CCDC125 in the molecular mechanisms related to cellular motility. Cell motility is an important function for many physiological processes, such as embryonic morphogenesis, wound healing, immune-cell trafficking, muscle contraction and neuronal network development (7). In the regulation and coordination of the cytoskeletal remodeling required for these events, RhoGTPase is known to play an important role. At present, 20 members of the RhoGTPase family have been identified in mammalian cells (8), and most attention has been directed towards three widely expressed members, RhoA, Rac1 and cdc42. These three proteins have unique and overlapping functions. In terms of cell migration 
and cytoskeletal organization, cdc 42 controls the assembly of filopodia $(9,10)$, Rac1 stimulates the formation of lamellipodia and membrane raffles (11), and RhoA regulates the assembly of stress fibers (12). Therefore, we studied the effect of Kenae/CCDC125 on the activity of the three molecules: RhoA, Rac1 and cdc42. This study demonstrated that Kenae/CCDC125 functions to deregulate the activity of all three molecules. These results suggest that Kenae/ CCDC125 is associated with cell motility through activation control of RhoA, Rac1 and cdc42.

\section{Materials and methods}

Antibodies and reagents. The anti-FLAG polyclonal antibody was purchased from Sigma. Anti-RhoA (26C4) and anticdc42 (B-8) were from Santa Cruz Biotechnology, and antiRac1 (clone 23A8) was from Upstate. 1-Oleoyl lysophosphatidic acid (sodium salt) (LPA) was from Cayman Chemical. Epidermal growth factor (EGF) and bradykinin were from Wako.

Gene cloning of an antigen recognized in the serum of an Isaac's syndrome patient. cDNA encoding Kenae/CCDC125 was obtained by immunoscreening using the serum antibody of an Isaac's syndrome patient expressing the gene of a human genome. Total RNAs were extracted from human neuroblastoma cells (NB-1) by an acid guanidine/phenol chloroform method, and mRNAs were separated by affinity chromatography using oligo(dT) beads. RNA was reverse transcribed, and double-stranded cDNA was ligated to the $\lambda$ ZAP vector (Stratagene Co., Ltd.) (NB-1 cDNA library). The immunoscreening using the serum of the patient was carried out using the picoBlue Immunoscreening Kit (Stratagene Co., Ltd.). A positive clone (phage) was converted into a plasmid pBluescript II SK(+) by a helper phage. The nucleotide sequence of a DNA inserted into the pBluescript II SK(+) was determined by the ABI PRISM 377 DNA Sequencing System (Perkin Elmer Co., Ltd.) based on a dye terminator method using M13 Primer M4 and M13 Primer RV (Takara). The Poly (A)+ chain was found by analysis from the $3^{\prime}$ terminal in the nucleotide sequence. Furthermore, we identified the first methionine by 5'-RACE (rapid amplification of cDNA ends) experiments (13) using the NB-1 cDNA library resulting in the cloning of a full-length Kenae/ CCDC125 cDNA.

Plasmid constructions. The coding sequence of the Kenae/ CCDC125 protein was inserted into the BamHI-EcoRV site of the pcDNA3-FLAG expression vector (FLAG-Kenae/ CCDC125). To construct GST-Rhotekin, the rhotekin Rhobinding domain coding region (18-89 amino acids) was amplified by PCR using primer set 5 '-cgeggatccagatggca ctcagcctggag-3' and 5'-cgcgaattcagcetgtcttctccagcacctg-3' with NIH3T3 cDNA. To construct GST-PAK, PAK1BRac1/cdc42 binding domain coding region (67-150 amino acids) was amplified by PCR using primer set 5'-cgcggatcc ttttaccgatccattttacctggagataaaac-3' and 5'-cgcgaattcaagacacag ccttcacattcaaggcattag-3' with HEK293 cDNA. These fragments were digested with $B a m \mathrm{HI} / E c o$ RI and subcloned into pGEX6P-1 (Amersham Bioscience).
Real-time RT-PCR experiments. Total RNA was isolated from the brain, heart, liver, lung, kidney, spleen, thymus, colon, intestine, testis, skin, eye, skeletal muscle, bone marrow and bone from male mice ( 8 weeks old) using Isogene (Nippon Gene). First-strand cDNA was synthesized with random hexamers and reverse transcriptase (ReverTraAce; Toyobo) using $1 \mu \mathrm{g}$ of total RNA in a $20-\mu 1$ reaction volume. Real-time PCR reactions were carried out using TaqMan Universal Master Mix (Applied Biosystems) and Universal Probe Library assays designed with the ProbeFinder software (Roche Applied Science). The primer sequences and Universal Probe numbers used are available upon request. ABI PRISM 7500 SDS was programmed to an initial step of 2 min at $50^{\circ} \mathrm{C}$ and $10 \mathrm{~min}$ at $95^{\circ} \mathrm{C}$, followed by 40 cycles of $15 \mathrm{sec}$ at $95^{\circ} \mathrm{C}$ and $1 \mathrm{~min}$ at $60^{\circ} \mathrm{C}$. The primers used were as follows: mouse Kenae/CCDC125, 5'-GCGAAACCAGTTTCCAGTATTC-3' (forward) and 5'-GCTCATCCGACAATTCTGAGT-3' (reverse) (probe no. 69) and 18s ribosomal RNA, 5'-AAATC AGTTATGGTTCCTTTGGTC-3' (forward) and 5'-GCTCTA GAATTACCACAGTTATCCAA-3' (reverse) (probe no. 55).

Cell establishment and culture. For the establishment of stable cell lines, HEK293 cells were seeded in 10-cm dishes before transfection. The cells were transfected with pcDNA3 containing the FLAG-Kenae/CCDC125 (4 $\mu \mathrm{g} /$ dish) using Fugene 6 (Roche) according to the manufacturer's instructions. Expressing cells were selected using a medium containing $1 \mathrm{mg} / \mathrm{ml} \mathrm{G} 418$ (Roche). Immunoblotting with Western blot analysis against FLAG-tag was used to quantitate FLAG-Kenae/CCDC125, and the cell line expressing a high level of FLAG-Kenae/CCDC125 (FLAGKenae/CCDC125/HEK293) was selected and used for the present study. These stable cell lines were maintained in MEM supplemented with 10\% fetal bovine serum (FBS), non-essential amino acids and penicillin/streptomycin at $37^{\circ} \mathrm{C}$ in $5 \% \mathrm{CO}_{2}$.

Immunofluorescence. HEK293 stable cell lines were plated onto glass coverslips. After $24 \mathrm{~h}$, cells were fixed, permeabilized and blocked. Cells were then incubated with the primary antibody, rabbit anti-FLAG, followed by staining with Alexa Fluor 488 anti-rabbit IgG second antibody (1:1000 dilution each; Molecular Probe).

Wound healing cell migration assay. Empty vector or FLAGKenae/CCDC125 stable-expressing HEK293 cell lines were plated onto collagen I-coated glass well plates (Iwaki) in MEM containing 10\% FBS. Twenty-four hours after plating, a confluent cell monolayer of each cell line was scraped with a 26-gauge needle, and the plate was then placed in a chamber fixed to the stage of a Leica AS MDW inverted microscope (Leica) and maintained at $37^{\circ} \mathrm{C}$ in an atmosphere containing $5 \% \mathrm{CO}_{2}$. Sequence time-lapse images were collected through a $\mathrm{x} 20$ objective and projected onto a CCD camera. Images were collected every $10 \mathrm{~min}$ for $4 \mathrm{~h}$.

RhoGTPase activity assays. HEK293 stable cell lines were seeded in 50-mm culture dishes and serum-starved for $24 \mathrm{~h}$. The cells were stimulated and lysed for $5 \mathrm{~min}$ in ice-cold cell lysis buffer [50 mM Tris/HCl (pH 7.5), $100 \mathrm{mM} \mathrm{NaCl}, 10 \mathrm{mM}$ 


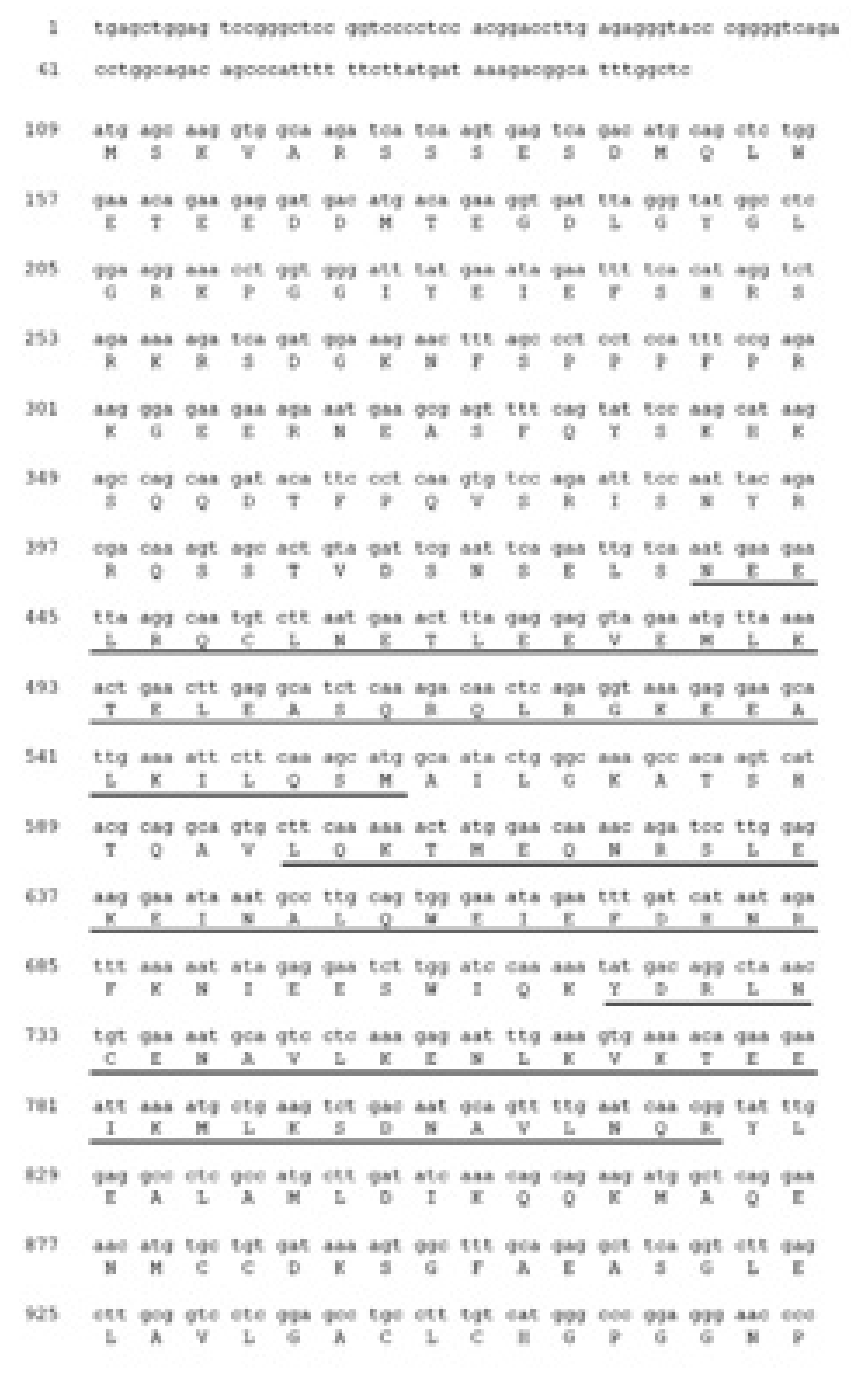

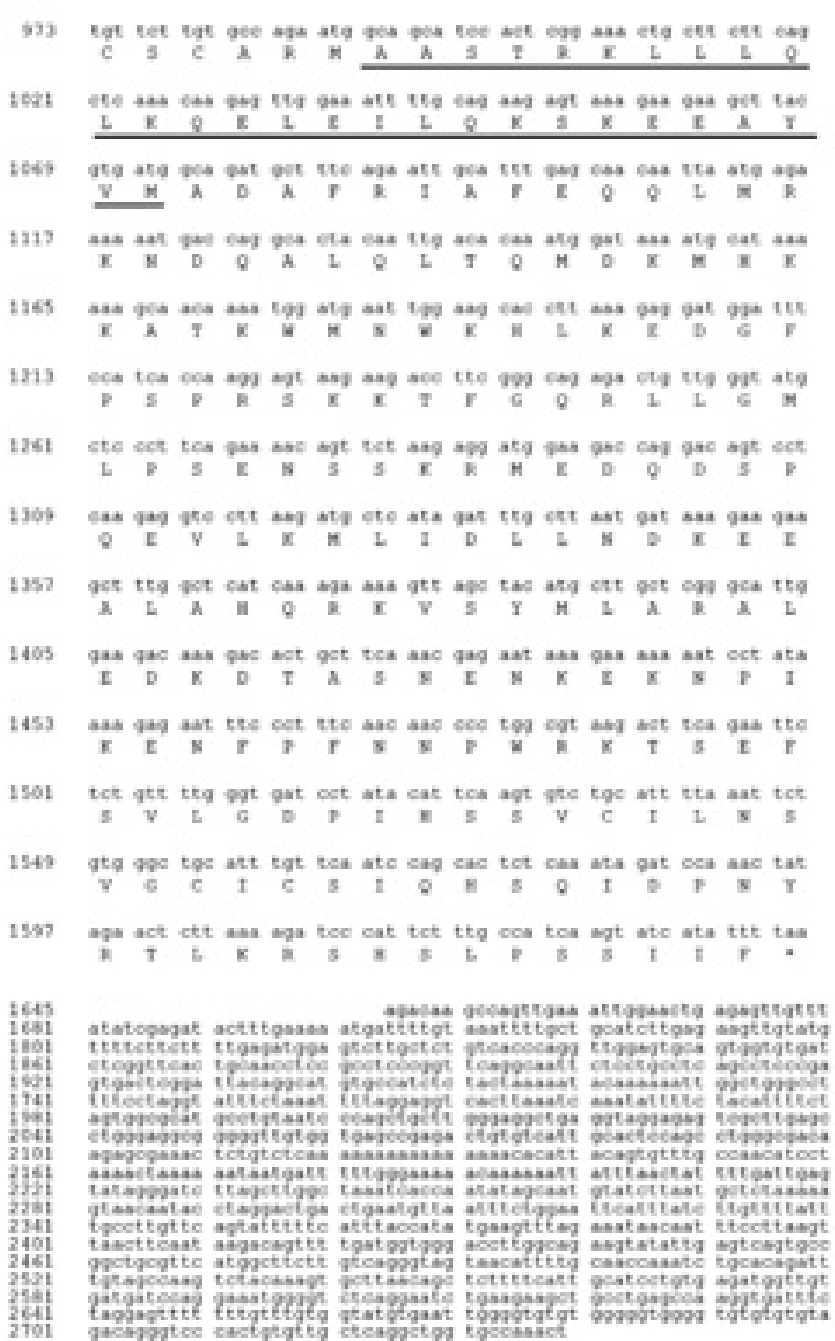

Figure 1. Identification of a novel gene Kenae/CCDC125. (A) cDNA and the predicted amino acid sequence of Kenae/CCDC125. Putative coiled-coil domains are underlined. Nucleotide sequence of full-length human Kenae/CCDC125 cDNA and deduced amino acid sequence of Kenae/CCDC125. This sequence has been submitted to GenBank under accession no. AB024691 (1999).

$\mathrm{MgCl}_{2}, 1 \%$ Triton X-100, $10 \mathrm{mM} \mathrm{NaF}, 1 \mathrm{mM} \mathrm{Na}_{3} \mathrm{VO}_{4}, 10 \mu \mathrm{g} /$ $\mathrm{ml}$ each of leupeptin and aprotinin and $1 \mathrm{mM}$ phenylmethylsulfonyl fluoride]. Cell lysates were clarified by centrifugation at $15,000 \mathrm{rpm}$ at $4^{\circ} \mathrm{C}$ for $5 \mathrm{~min}$. GTP-RhoA was precipitated from the remainder of the lysates using $60 \mu \mathrm{g}$ of GST-Rhotekin. GTP-Rac1 and GTP-cdc42 were precipitated from the remainder of the lysates using $4.5 \mu \mathrm{g}$ of GST-PAK for $30 \mathrm{~min}$ at $4^{\circ} \mathrm{C}$. After precipitation, the beads were washed two times with lysis buffer and wash buffer [25 mM Tris/ $\mathrm{HCl}$ (pH 7.5), $30 \mathrm{mM} \mathrm{MgCl}$ and $40 \mathrm{mM} \mathrm{NaCl}$. The bound proteins were analyzed by Western blotting using anti-RhoA, -Rac1 or -cdc42 antibodies.

\section{Results}

Gene cloning of an antigen recognized in the serum of a patient with Isaac's syndrome. To identify the molecules associated with Isaac's syndrome, we carried out an immunoscreening from the human neuroblastoma (NB-1) cell oligo(dT)-primed cDNA expression library using the serum of the patient. The positive clone contained a cDNA of $1347 \mathrm{bp}$ encoding an ORF of 386 amino acids with a poly-A tail. The 5 '-end was isolated from the same cDNA library by a 5'RACE (rapid amplification of cDNA ends) method (13). In Fig. 1, the composite nucleotide sequence of the cDNA and deduced amino acid sequence is shown. The 5'-untranslated nucleotide sequence contains $108 \mathrm{bp}$ and the 3'-untranslated region, $1095 \mathrm{bp}$. The cDNA sequence encodes a complete ORF of 511 amino acids and has four coiled-coil domains (Fig. 1 underlined). The nucleotide sequence was subjected to a homology search with GenBank. A similar sequence had not been reported, and it was found that the nucleotide sequence was a novel gene. We named this sequence 'Kenae' and registered it into the GenBank in 1999 (GenBank accession no. AB024691; Kawahara K, Arimura H, Arimura K, Soejima Y, Osame M, Maruyama I and Nakajima T). This gene can be referred to as NCBI Entrez Gene (Accession no. NM_176816, GeneID: 202243, gene name: CCDC125).

Expression profile of Kenae/CCDC125 mRNA. The coding sequence of mouse Kenae/CCDC125 is homologous with human Kenae/CCDC125 by $\geq 85 \%$ (NCBI Entrez Gene under 


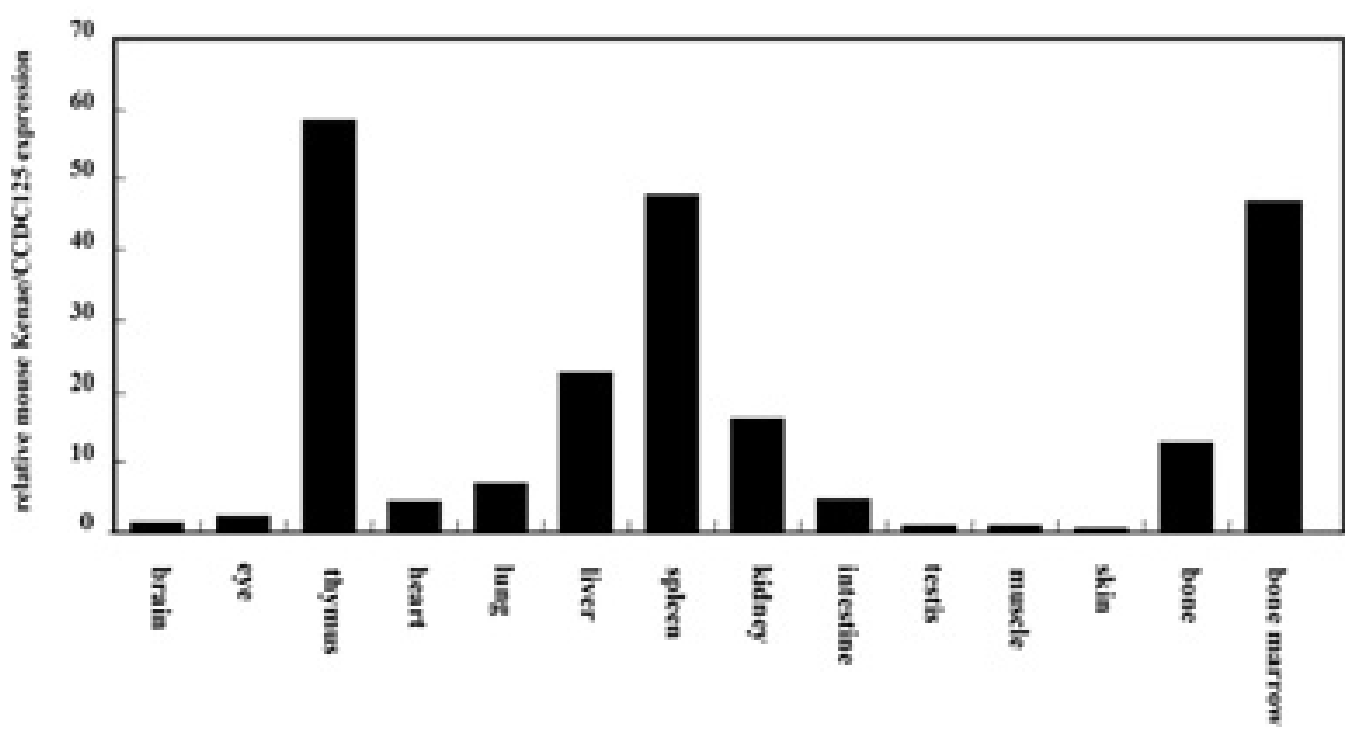

Figure 2. Expression profiles of Kenae/CCDC125. Mouse Kenae/CCDC125 mRNA detected by real-time RT-PCR in several mouse tissues. Expression data were normalized against the amount of 18 s ribosomal RNA PCR product.

Accession no. NM_183115, GeneID 76041). To confirm the expression of Kenae/CCDC125 in vivo, we prepared RNA from a variety of mouse tissues, including the brain, eye, thymus, heart, lung, liver, spleen, kidney, intestine, testis, skeletal muscle, skin, bone and bone marrow and analyzed the Kenae/CCDC125 mRNA expression in these tissues by real-time RT-PCR. Based on the cDNA sequence of mouse Kenae/CCDC125, we designed primers for real-time RTPCR to quantify the expression of Kenae/CCDC125 in different mouse tissues. 18S ribosomal RNA was used as a reference gene. As shown in Fig. 2, high expression of Kenae/ CCDC125 was demonstrated in the spleen, thymus and bone marrow, while low amounts of Kenae/CCDC125 mRNA were present in most tissues.

Association of Kenae/CCDC125 with cell motility. To elucidate the Kenae/CCDC125-mediated cellular function, we established the HEK293 cell lines stably expressing the FLAG-Kenae/CCDC125 (FLAG-Kenae/CCDC125/HEK293) or empty vector (vector/HEK293), respectively. Expression of FLAG-Kenae/CCDC125 protein in the cell clones was then detected by Western blot analysis using the anti-FLAG antibody. The molecular weight of Kenae/CCDC125 was found to be $\sim 65 \mathrm{kDa}$ (Fig. 3A). We further studied the localization of Kenae/CCDC125 protein in the cells by immunostaining using the anti-FLAG antibody. Stably expressed FLAG-Kenae/CCDC125 protein was localized in the cytoplasm (Fig. 3B). Next, to determine the phenotype of Kenae/CCDC125-expressing cells, we observed FLAGKenae/CCDC125/HEK293 stable cell lines under normal culture condition using a time-lapse microscope and compared them with the control cell line (vector/HEK293). As the motility of Kenae/CCDC125-expressing cells appreared to be diminished (data not shown), to confirm the motility of each cell line, we used the wound healing cell migration assay. A confluent cell layer of each cell line was scratched off, and cells were allowed to migrate into the empty space (Fig. 3C).
Images of the cells after scratching were captured immediately (Fig. 3C, panels a and c) and $4 \mathrm{~h}$ later (Fig. 3C, panels $b$ and d). The migrated distance of each cell line was measured every hour (Fig. 3D). The migration speed of the FLAG-Kenae/CCDC125/HEK293 cells was significantly slower than that of the vector/HEK293 cells. This indicated that the consequent expression of Kenae/CCDC125 represses cellular motility.

Deregulation of RhoGTPase by Kenae/CCDC125. RhoA/ Rac1/cdc42, members of the RhoGTPase family, regulate cell motility through actin cytoskeletal reorganization in response to extracellular stimulation such as LPA, EGF and bradykinin (14). As Kenae/CCDC125 was demonstrated to delay cell motility (Fig. 3), it was important to study the effect of Kenae/CCDC125 on RhoA, Rac1 and cdc42 activities. To assess the effect of Kenae/CCDC125 on RhoA, Rac 1 and cdc42 activities, we examined the amount of activated RhoA, Rac 1 and cdc42 in the FLAG-Kenae/ CCDC125/HEK293 stable cells after stimulation by LPA, EGF or bradykinin, respectively. We performed GST pulldown assays to measure the active form of GTP-bound RhoGTPase using the rhotekin Rho-binding domain (15) or the PAK1B-Rac1/cdc42 binding domain fused to GST $(16,17)$. After $24 \mathrm{~h}$ of serum deprivation, each stable cell line was treated with LPA, EGF or bradykinin for 1 or $3 \mathrm{~min}$, and the active form of GTP-bound RhoA, Rac1 or cdc42 was isolated from cell lysates by GST pulldown (Fig. 4). The proteins were detected by immunoblotting using specific antibodies, and the amount of protein of each band was quantified using densitometry (ImageJ software). As shown in Fig. 4, the active form of RhoA, Rac1 or cdc42 was barely detectable in unstimulated vector/HEK293 cells. However, in the FLAG-Kenae/CCDC125/HEK293 cells, the active form of RhoA, Rac1 or cdc42 proteins was detectable even under unstimulated conditions. After extracellular stimulation, the RhoA activity was increased 14.0-fold in vector/HEK293 

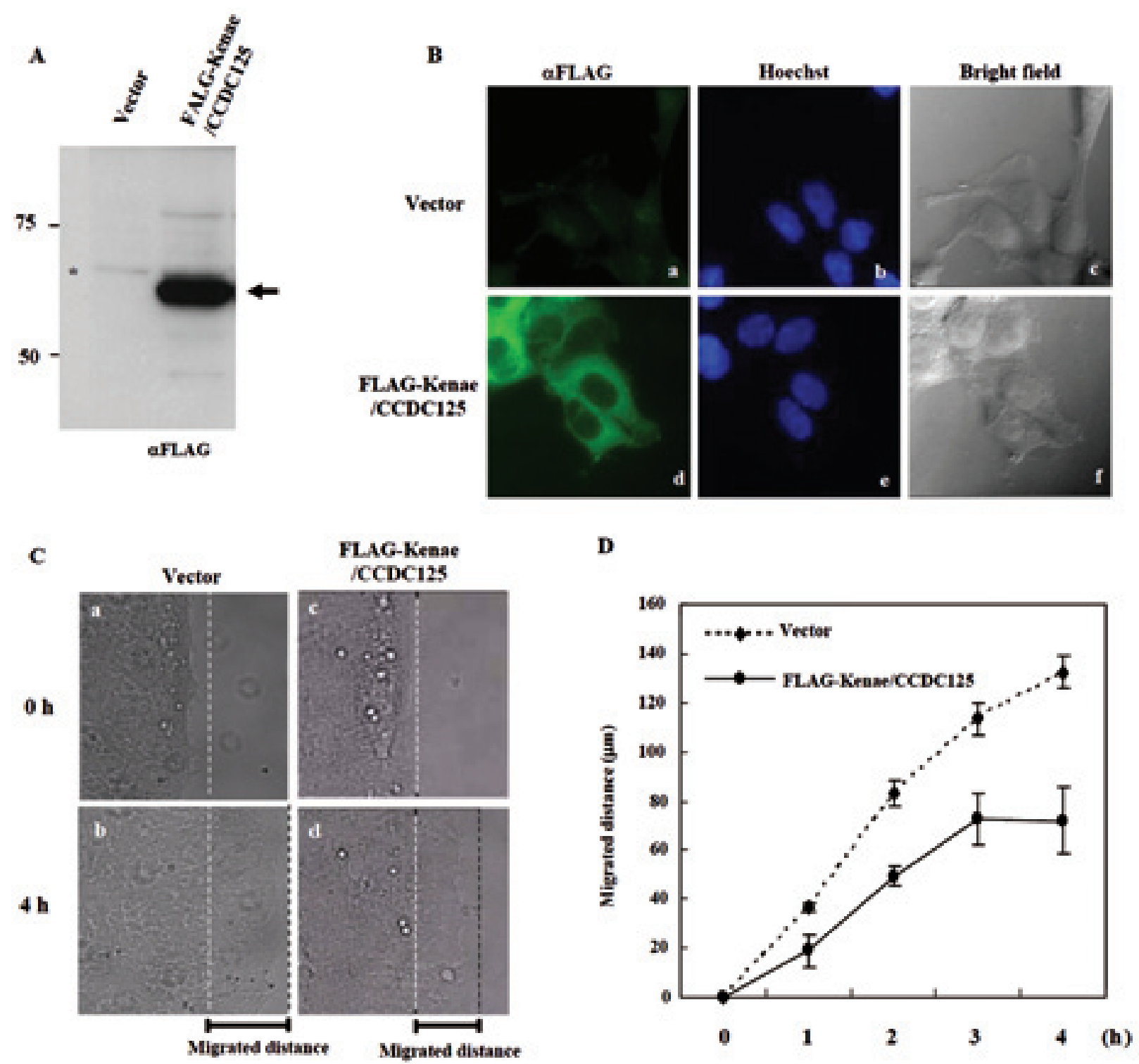

Figure 3. Establishment and characterization of Kenae/CCDC125 stable expressed cell line. (A) Immunoblot analysis of Kenae/CCDC125 protein. Each whole cell lysate extracted from HEK293 (lane 1) or FLAG-Kenae/CCDC125/HEK293 (lane 2) cell lines was detected by immunoblotting with anti-FLAG antibody. Arrow, FLAG-Kenae/CCDC125 protein; asterisk, the non-specific band. (B) Cytoplasmic localization of Kenae/CCDC125 in HEK 293 cells. Empty vector or FLAG-Kenae/CCDC125-expressing HEK293 cell lines were stained with anti-FLAG antibody (a and d) and DAPI (b and e). Whole cell images (c and f). (C) Effect of Kenae/CCDC125 expression on the healing of scratch wounds in confluent cells. The wound-filling capacity of both HEK293 stable cell lines was monitored by time-lapse microscopy for $4 \mathrm{~h}$ under normal culture condition. Confluent monolayers of cells were scraped to create a clear area (0 h, a and c). After 4 h, the wound closure was monitored (b and d). (D) The migrated distance of vector or FLAG-Kenae/ CCDC125/HEK293 cell lines. Data from three randomly selected fields were presented as the means \pm SE every hour for 4 hours.

cells and 1.6-fold in FLAG-Kenae/CCDC125/HEK293 cells (Fig. 4A). The Rac1 activity was elevated 2.3-fold in the vector/HEK293 cells and 1.4-fold in the FLAG-Kenae/ CCDC125/HEK293 cells (Fig. 4B). The cdc42 activity was increased 3.3-fold in the vector/HEK293 cells and 1.2-fold in the FLAG-Kenae/CCDC125/HEK293 cells (Fig. 4C). Thus, in the FLAG-Kenae/CCDC125/HEK293 cells, the active form of RhoA/Rac1/cdc42 proteins was detectable even under unstimulated conditions, and the activation of RhoA/Rac1/cdc42 proteins was poor after stimulation. These results suggest that Kenae/CCDC125 induces both constitutive activation of RhoGTPase and unresponsiveness of RhoGTPase to extracellular stimulation.

\section{Discussion}

Isaac's syndrome has been recognized as an autoimmune disease. Recently, it has been reported that, not all, but some Isaac's syndrome patients develop auto-antibodies to VGKCs (4). However, the underlying mechanisms of this disease are not fully understood. In this study, by immunoscreening using serum of a patient with Isaac's syndrome, we identified a novel gene named Kenae/CCDC125 (GenBank 1999) (Fig. 1). The amino-acid sequence analysis of Kenae/ CCDC125 implied that it forms four coiled-coil structures. The coiled-coil structure is known to be involved in diverse organizational and regulatory processes in eukaryotic cells. 
$\mathbf{A}$
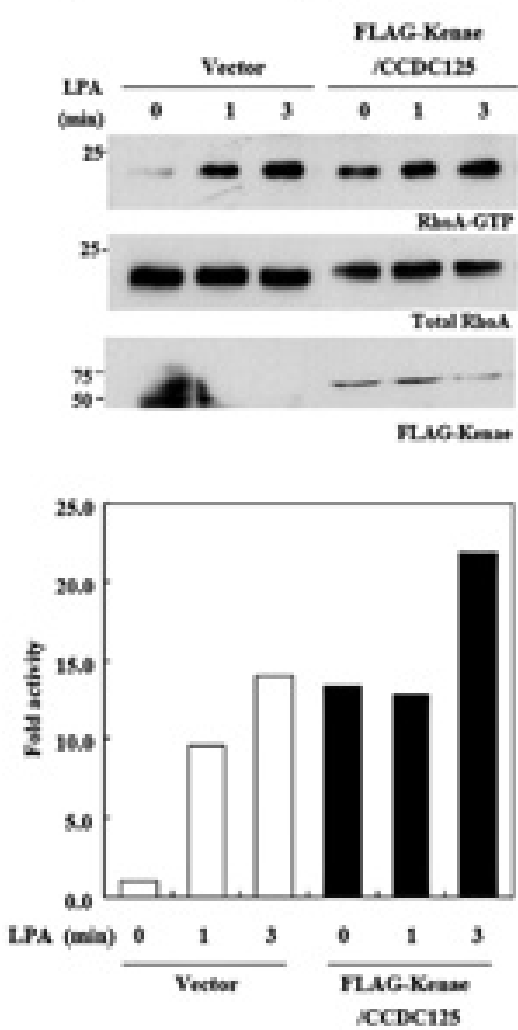

B
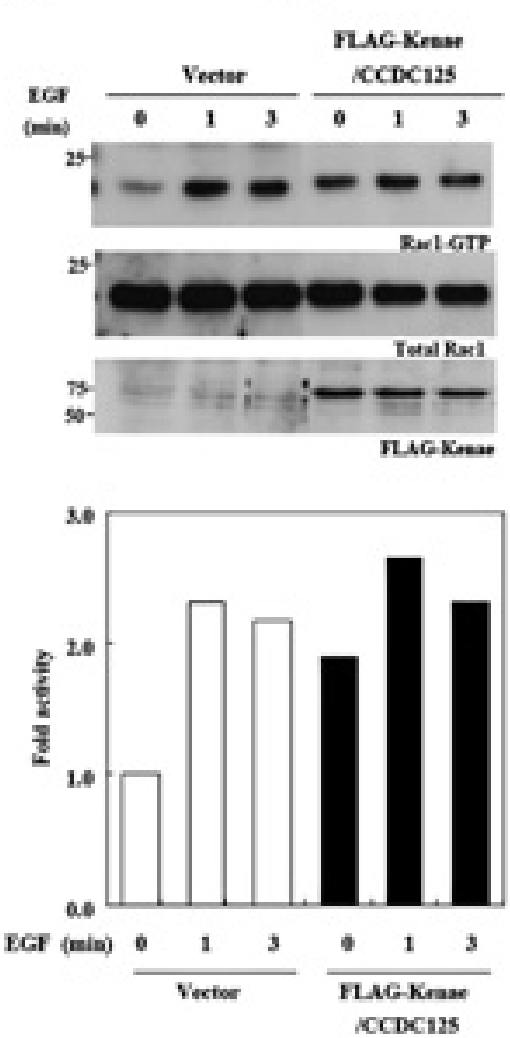

c
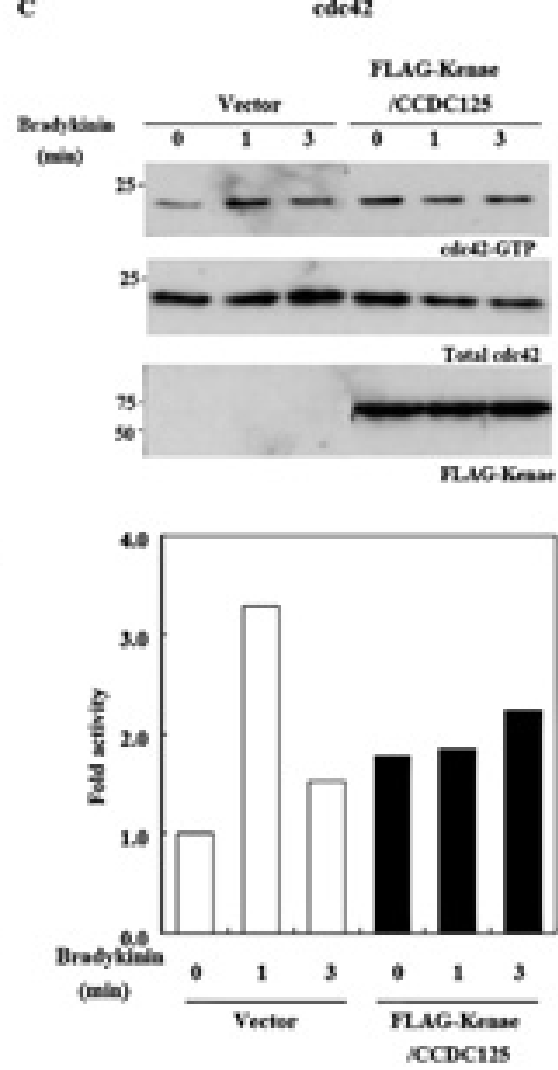

Figure 4. Effect of Kenae/CCDC125 on the activity of RhoGTPase, RhoA/Rac1/cdc42. (A-C) Representative experiment showing the amount of active GTPbound RhoA (A), Rac1 (B) or cdc42 (C) determined by a GST pulldown assay, from non-stimulated or stimulated [5 $\mu \mathrm{M}$ LPA (A), $100 \mathrm{ng} / \mathrm{ml}$ EGF (B) or $1 \mu \mathrm{M}$ Bradykinin (C)] vector or FLAG-Kenae/CCDC125/HEK293 stable cell lines for the indicated time. GTP-bound Rho family protein levels were determined by immunoblotting with specific antibodies following the pulldown (upper panels) or in the total cell extracts (middle panels). Stable expressed FLAGKenae/CCDC125 was detected using anti-FLAG antibody (bottom panels). (A-C graphs) The Rho-GTP pulldown results were analyzed and indicated as fold activity. The amount of the active form of the GTP-bound molecule was divided by the total amount of each RhoGTPase, and the fold activity of each time point was calculated by defining one at time zero in vector or FLAG-Kenae/CCDC125/HEK293 stable cell line, respectively. These results were confirmed in three independent experiments.

They provide cables and networks in the cyto- and nucleoskeleton molecular scaffolds. The dynamic coiled-coil formation can play a central role in the generation of conformational changes resulting in dramatic movements of one part of a protein to another. Mutations in coiled-coil proteins have been implicated in a wide variety of human diseases such as severe skin fragility, muscular dystrophies, neurodegenerative diseases, progeria and cancer (18-25). Furthermore, by searching the database of the National Center for Biotechnology Information, homologous sequences of Kenae/CCDC125 were found to be present in Mus musculus (NM_183115), Rattus norvegicus (XM_574843), Danio rerio (GeneID: 100048918), Gallus gallus (XM_424893), Bos Taurus (XM_593329), Pan troglodytes (XM_001145441) and Canis lupus familiaris (XM_544367, XM_856747). Thus, this gene is conserved only in vertebrates, suggesting that Kenae/ CCDC125 has been recently generated with evolution. Realtime PCR analysis in the mouse revealed that the Kenae/ CCDC125 gene was highly expressed in lymphoid tissues such as the thymus, spleen and bone marrow (Fig. 2). This partial expression in vivo suggests that this gene is not a housekeeping gene encoding cellular structure proteins common to all cell types. Instead, it may have a specific function required for limited cell types and/or for a specific stage of development. These results suggest that Kenae/ CCDC125 may play an important role in the cell components of lymphoid tissues in vertebrates.

To address the cellular function of Kenae/CCDC125, we established stable FLAG-tagged Kenae/CCDC125-expressing HEK 293 cells. Kenae/CCDC125 protein $(\sim 65 \mathrm{kDa})$ was localized in the cytoplasm and importantly, Kenae/CCDC125 expression decreased the cell motility in HEK293 cells (Fig. 3). Animal cell movement is affected through a combination of protrusive and contractile events regulated by the remodeling of actin cytoskeleton (26). Members of the RhoGTPase family, including RhoA, Rac1 and cdc42 are known to play an important role in the regulation and coordination of the actin cytoskeleton (14). These factors act as molecular switches between the inactive GDP-bound form and the active GTP-bound form (8). In this study, we found that Kenae/CCDC125 deregulated the activation of RhoA, Rac1 and cdc42 (Fig. 4), suggesting that Kenae/CCDC125 controls cell motility through the regulation of RhoA, Rac1 and cdc42 activity. Therefore, it was of interest whether Kenae/CCDC125 interacts with RhoA, Rac1 or cdc42. However, the direct binding of Kenae/CCDC125 to RhoA, Rac1 or cdc42 was not observed by GST pulldown and immunoprecipitation assays (data not shown). These results 
suggest that Kenae/CCDC125 may interact with the molecules associated with the activation process of RhoGTPase. The function of RhoGTPase has been reported to be regulated by the switching between the inactive and the active form (8). This switching is controlled by RhoGTPase family-specific guanine nucleotide exchange factors (GEFs) (27) and GTPase-activating proteins (GAPs) (28). Therefore, we are currently investigating the relationship between Kenae/ CCDC125 and GEF or GAP molecules.

In conclusion, using the serum of an Isaac's syndrome patient, we cloned a novel gene named Kenae/CCDC125 which has four coiled-coil domains. Kenae/CCDC125 was mainly expressed in lymphoid tissues. Furthermore, reduced cell motility by Kenae/CCDC125 expression was demonstrated through the regulation of RhoGTPase. These results suggest that Kenae/CCDC125 may play an important role in controling the cell motility of immune systems. Cell motility is vital for other physiological processes, such as morphogenesis, wound healing, muscle contraction and neuronal network development. Therefore, further studies involving the Kenae/CCDC125 gene may provide new insight into the molecular mechanisms of these physiological processes.

\section{Acknowledgements}

This study was supported, in part, by the National Institute of Biomedical Innovation; the Japanese Ministry of Education, Culture, Sports, Science and Technology; the Japanese Ministry of Health, Labour and Welfare; the Kato Memorial Trust for Nanbyo Research; the Japan Medical Association; Nagao Memorial Fund; Kanae Foundation for Life and Socio-Medical Science; Japan Research Foundation for Clinical Pharmacology; Kanagawa Nanbyo Foundation; Kanagawa Academy of Science; Technology Research Grants; Japan College of Rheumatology; the Nakajima Foundation; Osaka Foundation for Cancer Research; Japan Society for the Promotion of Science, New Energy and Industrial Technology Development Organization; Mochida Pharmaceutical Co. Ltd.; the Kanagawa High-Technology Foundation; Kanto Bureau of Economy, Trade and Industry; Mitsui Life Insurance Co., Ltd.; the Uehara Memorial Foundation; the Takeda Science Foundation; Heiwa Nakajima Foundation; the Sagawa Foundation for Promotion of Cancer Research; the Tokyo Biochemical Research Foundation, the Kanagawa High-Technology Foundation and 1 st Inoue Foundation for Science and Japan Society for the Promotion of Science.

\section{References}

1. Isaacs H: A syndrome of continuous muscle-fiber activity. J Neurol Neurosurg Psychiatry 24: 319-325, 1961.

2. Kimura J: Neuromuscular Diseases Characterized by Abnormal Muscle Activity. Oxford University Press, USA, 1983.

3. Newsom-Davis J and Mills KR: Immunological associations of acquired neuromyotonia (Isaacs' syndrome). Report of five cases and literature review. Brain 116: 453-469, 1993.

4. Shillito P, Molenaar PC, Vincent A, Leys K, Zheng W, van den Berg RJ, Plomp JJ, van Kempen GT, Chauplannaz G, Wintzen AR, et al: Acquired neuromyotonia: evidence for autoantibodies directed against $\mathrm{K}^{+}$channels of peripheral nerves. Ann Neurol 38: 714-722, 1995.
5. Arimura K, Watanabe O, Kitajima I, Suehara M, Minato S, Sonoda Y, Higuchi I, Takenaga S, Maruyama I and Osame M: Antibodies to potassium channels of PC12 in serum of Isaacs' syndrome: Western blot and immunohistochemical studies. Muscle Nerve 20: 299-305, 1997.

6. Hart IK, Waters C, Vincent A, Newland C, Beeson D, Pongs O, Morris C and Newsom-Davis J: Autoantibodies detected to expressed $\mathrm{K}^{+}$channels are implicated in neuromyotonia. Ann Neurol 41: 238-246, 1997.

7. Singer SJ and Kupfer A: The directed migration of eukaryotic cells. Annu Rev Cell Biol 2: 337-365, 1986.

8. Etienne-Manneville S and Hall A: Rho GTPases in cell biology. Nature 420: 629-635, 2002.

9. Kozma R, Ahmed S, Best A and Lim L: The Ras-related protein $\mathrm{Cdc} 42 \mathrm{Hs}$ and bradykinin promote formation of peripheral actin microspikes and filopodia in Swiss 3T3 fibroblasts. Mol Cell Biol 15: 1942-1952, 1995.

10. Nobes CD and Hall A: Rho, rac, and cdc42 GTPases regulate the assembly of multimolecular focal complexes associated with actin stress fibers, lamellipodia, and filopodia. Cell 81: 53-62, 1995.

11. Ridley AJ and Hall A: The small GTP-binding protein rho regulates the assembly of focal adhesions and actin stress fibers in response to growth factors. Cell 70: 389-399, 1992.

12. Ridley AJ and Hall A: Distinct patterns of actin organization regulated by the small GTP-binding proteins Rac and Rho. Cold Spring Harb Symp Quant Biol 57: 661-671, 1992.

13. Frohman MA, Dush MK and Martin GR: Rapid production of full-length cDNAs from rare transcripts: amplification using a single gene-specific oligonucleotide primer. Proc Natl Acad Sci USA 85: 8998-9002, 1988.

14. Hall A: Rho GTPases and the actin cytoskeleton. Science 279: 509-514, 1998.

15. Reid T, Furuyashiki T, Ishizaki T, Watanabe G, Watanabe N, Fujisawa K, Morii N, Madaule P and Narumiya S: Rhotekin, a new putative target for Rho bearing homology to a serine/ threonine kinase, PKN, and rhophilin in the rho-binding domain. J Biol Chem 271: 13556-13560, 1996.

16. Sander EE, van Delft $S$, ten Klooster JP, Reid T, van der Kammen RA, Michiels F and Collard JG: Matrix-dependent Tiam1/Rac signaling in epithelial cells promotes either cell-cell adhesion or cell migration and is regulated by phosphatidylinositol 3-kinase. J Cell Biol 143: 1385-1398, 1998.

17. Matsuo N, Hoshino M, Yoshizawa M and Nabeshima $Y$ : Characterization of STEF, a guanine nucleotide exchange factor for Rac1, required for neurite growth. J Biol Chem 277: 2860-2868, 2002

18. Magin TM, Reichelt $\mathrm{J}$ and Hatzfeld M: Emerging functions: diseases and animal models reshape our view of the cytoskeleton. Exp Cell Res 301: 91-102, 2004.

19. Mounkes L, Kozlov S, Burke B and Stewart CL: The laminopathies: nuclear structure meets disease. Curr Opin Genet Dev 13: 223-230, 2003.

20. Puls I, Jonnakuty C, LaMonte BH, Holzbaur EL, Tokito M, Mann E, Floeter MK, Bidus K, Drayna D, Oh SJ, Brown RH Jr, Ludlow CL and Fischbeck KH: Mutant dynactin in motor neuron disease. Nat Genet 33: 455-456, 2003.

21. Hirokawa N and Takemura R: Molecular motors in neuronal development, intracellular transport and diseases. Curr Opin Neurobiol 14: 564-573, 2004.

22. Chigira S, Sugita K, Kita K, Sugaya S, Arase Y, Ichinose M, Shirasawa $\mathrm{H}$ and Suzuki N: Increased expression of the Huntingtin interacting protein-1 gene in cells from Hutchinson Gilford Syndrome (Progeria) patients and aged donors. J Gerontol A Biol Sci Med Sci 58: B873-B878, 2003.

23. Mounkes LC and Stewart CL: Aging and nuclear organization: lamins and progeria. Curr Opin Cell Biol 16: 322-327, 2004.

24. Raff JW: Centrosomes and cancer: lessons from a TACC. Trends Cell Biol 12: 222-225, 2002.

25. McClatchey AI: Merlin and ERM proteins: unappreciated roles in cancer development? Nat Rev Cancer 3: 877-883, 2003.

26. Pellegrin S and Mellor H: Actin stress fibres. J Cell Sci 120: 3491-3499, 2007.

27. Zheng Y: Dbl family guanine nucleotide exchange factors. Trends Biochem Sci 26: 724-732, 2001.

28. Moon SY and Zheng Y: Rho GTPase-activating proteins in cell regulation. Trends Cell Biol 13: 13-22, 2003. 